\title{
Collimation method using a double grating system
}

\author{
Luis Miguel Sanchez-Brea, ${ }^{1, *}$ Francisco Jose Torcal-Milla, ${ }^{1}$ Francisco Javier Salgado- \\ Remacha, ${ }^{1}$ Tomás Morlanes, ${ }^{2}$ Isidoro Jimenez-Castillo, ${ }^{1}$ and Eusebio Bernabeu ${ }^{1}$ \\ ${ }^{1}$ Universidad Complutense de Madrid, Applied Optics Complutense Group, Optics Department, \\ Facultad de Ciencias Físicas, Ciudad Universitaria s.n., 28040, Madrid, Spain \\ ${ }^{2}$ AOTEK, Barrio de San Andrés, s.n., 28040 Mondragón, Guipúzcoa Spain \\ ${ }^{\star}$ Corresponding author: sanchezbrea@fis.ucm.es
}

Received 11 March 2010; revised 13 May 2010; accepted 18 May 2010; posted 19 May 2010 (Doc. ID 125355); published 8 June 2010

\begin{abstract}
We present a collimation technique based on a double grating system to locate with high accuracy an emitter in the focal plane of a lens. Talbot self-images are projected onto the second grating producing moiré interferences. By means of two photodetectors positioned just behind the second grating, it is possible to determine the optimal position of the light source for collimation by measuring the phase shift between the signals over the two photodetectors. We obtain mathematical expressions of the signal in terms of defocus. This allows us to perform an automated technique for collimation. In addition, a simple and accurate visual criterion for collimating a light source using a lens is proposed. Experimental results that corroborate the proposed technique are also presented. () 2010 Optical Society of America

OCIS codes: $\quad 050.0050,050.2770,120.1680$.
\end{abstract}

\section{Introduction}

Because of their long range of applications, double grating systems have been studied for many years [1-3]. These systems are found in several different devices such as optical encoders, machine tools, robotics, interferometers, and spectrometers [4-6]. Also, double grating systems have been successfully employed in wavefront sensing and beam aberration measurements [7-12]. Related to these applications, collimation testing appears as a useful application of double grating systems [13-15].

A way to obtain a collimated beam is to place a narrow light source, such as a LED, in the focal plane of a lens. The usual collimation technique based on double grating imaging uses Talbot self-imaging magnification [16]. When a collimated beam passes through a diffraction grating, self-images of the grating appear in the near field at certain distances, known as Talbot distances, which are $z_{T}=n p^{2} / \lambda$, where $p$ is the period of the grating, $\lambda$ is the wavelength, and $n$ is an integer. On the other hand, when the light beam is not colli-

0003-6935/10/173363-06\$15.00/0

(C) 2010 Optical Society of America mated, the Talbot distances change [17-19]. In both cases moiré fringes can be obtained when a second grating with the same period as the first is placed at some Talbot plane. Consequently, a way to test the collimation degree using a double grating system is to place the second grating at the location of the selfimages. Then the beam collimation is varied until the amplitude or contrast of the fringes formed after the second grating reaches a maximum. Although this technique leads to an easy collimation test, the resolution of the technique is low since it depends on the width of the self-images along $z$.

Here we propose an accurate collimation technique based on double grating imaging in which the measurement is performed to determine the phase shift between two optical signals captured after the second grating with two photodetectors laterally displaced with respect to the optical axis. The relative phase between both signals strongly depends on the degree of collimation of the beam. As a result, the accuracy of this technique is very high, as we demonstrate by experimental and theoretical results. An electronic circuit can measure the phase shift between both signals and therefore the proposed technique can be implemented in an automated fashion. Also, the collima- 
tion can be easily performed using a visual technique by means of the Lissajous figure obtained with both signals.

In Section 2 we use a near-field approach to obtain the intensity distribution of a double grating system (moiré system) when the emitter is not at the focus of the collimating lens. Analytical expressions are obtained that allow us to relate the phase shift of the signals and the defocus. In Section 3 we analyze the Lissajous figures obtained for different defocus degrees so that we propose a simple visual collimating test. Finally, in Section 4 an experimental demonstration of the system behavior is presented comparing it with the analytical results and corroborating its validity. For a standard configuration, a defocus of $1 \mu \mathrm{m}$ can be detected with the proposed technique.

\section{Noncollimated Beams through a Double Grating System}

Let us consider the double grating system in a onedimensional approach as shown in Fig. 1(a). For simplicity, let us consider a one-dimensional approach. A punctual and monochromatic light source with wavelength $\lambda$ is placed at a distance $z_{0}$ from an aberrationfree collimating lens, $L_{1}$, whose focal length is $f$. Then the light beam propagates a distance $z_{1}$ to the first diffraction grating $G_{2}$ and a distance $z_{2}$ from the first to the second grating $G_{3}$. The periods of both gratings are the same, $p_{2}=p_{3}=p$. The distance between $G_{2}$ and $G_{3}, z_{2}$, should be a multiple of the Talbot distance, $z_{T}=p^{2} / \lambda$, to obtain fringes with high contrast just behind the second grating. Henceforth, subindices 0,1 , 2 , and 3 refer to the light source, the lens, the first grating, and the second grating, respectively. Two photodetectors are positioned behind the second grating to convert the optical signals into electrical signals. Both photodetectors are symmetrically located with respect to the $z$ axis, over the same $x-y$ plane. In Fig. 1(a) the system is shown when the emitter is at the focal point of the lens $\left(z_{0}=f\right)$. In contrast, when the emitter is out of focus, as in Fig. 1(b), the beam is not collimated, and $z_{0}=f+\Delta z$, with $\Delta z$ denoting a small displacement along the $z$ axis with respect to the focal plane.

The transmittance of gratings $G_{2}$ and $G_{3}$ is described by means of their Fourier series expansion as

$$
\begin{aligned}
& t_{2}(x)=\sum_{n} a_{n} \exp \left[\operatorname{iqn}\left(x-\Delta x_{2}\right)\right], \\
& t_{3}(x)=\sum_{m} b_{m} \exp \left[\operatorname{iqm}\left(x-\Delta x_{3}\right)\right],
\end{aligned}
$$

where $q=2 \pi / p, a_{n}$ and $b_{m}$ are the Fourier coefficients of both gratings, $\Delta x_{2}$ and $\Delta x_{3}$ represent the relative displacement of the gratings with respect to the origin, and $n$ and $m$ are integers. We assume that $p$ is much larger than the wavelength so that a scalar approach can be used. The field in front of collimating lens $L_{1}$ can be obtained using a one-dimensional Fresnel approximation, resulting in (a)

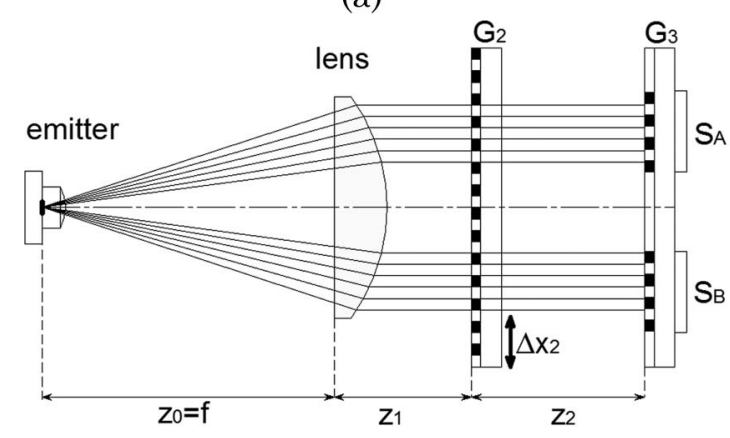

(b)

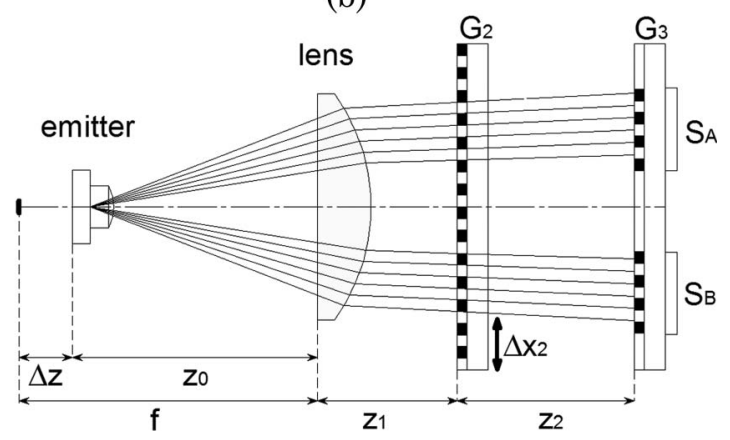

Fig. 1. Double grating collimating system with the light source (a) located at the focal point of lens $z_{0}=f$ and (b) placed at $z_{0}=f \pm \Delta z$.

$$
U_{1}\left(x_{1}\right)=U_{0} \frac{e^{i k(f+\Delta z)}}{\sqrt{f+\Delta z}} e^{i_{2(f+\Delta z)} x_{1}^{2}}
$$

where $k=2 \pi / \lambda$ and $U_{0}$ is the amplitude of the light field. Just behind the lens, defined as $h_{l}(x)=$ $\exp \left(-i k x^{2} / 2 f\right)$, the field results in $U_{1}^{\prime}\left(x_{1}\right)=U_{1}\left(x_{1}\right)$ $h_{l}\left(x_{1}\right)$. Since the defocus distance $\Delta z$ is usually much smaller than the focal length of the lens, $\Delta z \ll f$, we can approximate the complex amplitude just after the collimating lens as

$$
U_{1}^{\prime}\left(x_{1}\right) \approx U_{0} \frac{e^{i k(f+\Delta z)}}{\sqrt{f}} \exp \left(-i \frac{k \Delta z}{2 f^{2}} x_{1}^{2}\right)
$$

Equation (3) describes the propagation of a spherical wave except when $\Delta z=0$ results as a plane wave. When this wave propagates a distance $z_{1}$, the complex amplitude results:

$$
\begin{aligned}
U_{2}\left(x_{2}\right) & =\frac{e^{i k z_{1}}}{i \sqrt{\lambda z}} \int U_{1}^{\prime}\left(x_{1}\right) e^{i \frac{k}{2 z_{1}}\left(x_{2}-x_{1}\right)^{2}} \mathrm{~d} x_{1} \\
& \approx U_{0} \frac{e^{i k\left(f+\Delta z+z_{1}\right)}}{\sqrt{i f}} e^{i k \alpha x_{2}^{2} / 2},
\end{aligned}
$$

where $\alpha=1 / z_{1}-1 /\left(z_{1}-z_{1}^{2} \cdot \Delta z / f^{2}\right) \simeq-\Delta z / f^{2}$. Now, using Eqs. (4) and (1), we propagate the wave through the first grating $U_{2}^{\prime}\left(x_{2}\right)=U_{2}\left(x_{2}\right) t_{2}\left(x_{2}\right)$, followed by a distance $z_{2}$ to the second grating, resulting in 


$$
\begin{aligned}
U_{3}\left(x_{3}\right)= & \frac{e^{i k z_{3}}}{i \sqrt{\lambda z_{3}}} \int U_{2}^{\prime}\left(x_{2}\right) e^{i \frac{k}{2 z_{2}}\left(x_{3}-x_{2}\right)^{2}} \mathrm{~d} x_{2} \\
= & U_{0} \frac{e^{i k\left(\Delta z+z_{1}+z_{2}\right)}}{i \sqrt{f}} e^{i \frac{k}{2 z_{2}} x_{3 \frac{1}{2}+1 / \alpha z_{2}}} \\
& \times \sum_{n} a_{n} e^{-i q n \Delta x_{2}} e^{-i \frac{q^{2} n^{2} z_{2}}{2 k} 1+\alpha z_{2}} e^{i \frac{q n x_{3}}{1+\alpha z_{2}}}
\end{aligned}
$$

Thus we can write the intensity as

$$
\begin{aligned}
I_{3}\left(x_{3}\right)= & U_{3}\left(x_{3}\right) U_{3}^{*}\left(x_{3}\right)=\frac{I_{0}}{f} \sum_{n} \sum_{n^{\prime}} a_{n} a_{n^{\prime}} \\
& \times e^{-i q\left(n-n^{\prime}\right) \Delta x_{2}} e^{i \frac{q}{1+\alpha z_{2}}\left(n-n^{\prime}\right) x_{3}} e^{-i^{\frac{q^{2}}{2 k}\left(n^{2}-n^{\prime 2}\right)} \frac{z_{2}}{1+\alpha z_{2}}},
\end{aligned}
$$

with $I_{0}=U_{0} U_{0}^{*}$. The first exponential is related to the phase shift produced by the movement of grating $G_{2}$, the second exponential is related to the period of selfimages, and the last exponential is related to changes in the position of the Talbot planes.

The intensity distribution just behind the second grating, $I_{3}^{\prime}\left(x_{3}\right)=I_{3}\left(x_{3}\right) t_{3}\left(x_{3}\right)$, is given by

$$
\begin{aligned}
I_{3}^{\prime}\left(x_{3}\right)= & \frac{I_{0}}{f^{\prime}} \sum_{n} \sum_{n^{\prime}} \sum_{m} a_{n} a_{n^{\prime}}^{*} b_{m} e^{-i q\left[\left(n-n^{\prime}\right) \Delta x_{2}+m \Delta x_{3}\right]} \\
& \times e^{-i \frac{q^{2}}{2 k^{2}}\left(n^{2}-n^{\prime 2}\right) \frac{z_{2}}{1+\alpha z_{2}}} i q\left[\frac{\left(n-n^{\prime}\right)}{1+\alpha z_{2}}+m\right] x_{3} .
\end{aligned}
$$

Behind the second grating, a pair of photodetectors is used to transform the optical signals into electrical signals. The detectors have area size $w$ and their centers are placed at $x_{a}$ and $-x_{a}$. Signals $S_{A}$ and $S_{B}$ over the detectors are defined as an integration of $I_{3}^{\prime}\left(x_{3}\right)$ over the area of the photodetector:

$$
\begin{aligned}
S_{A}\left(\Delta x_{2}\right) & =\int_{-x_{a}-w / 2}^{-x_{a}+w / 2} I_{3}^{\prime}\left(x_{3}\right) \mathrm{d} x_{3}, \\
S_{B}\left(\Delta x_{2}\right) & =\int_{x_{a}-w / 2}^{x_{a}+w / 2} I_{3}^{\prime}\left(x_{3}\right) \mathrm{d} x_{3},
\end{aligned}
$$

which result in

$$
\begin{aligned}
S_{A, B}\left(\Delta x_{2}\right)= & \frac{I_{0}}{f} \sum_{n} \sum_{n^{\prime}} \sum_{m} a_{n} a_{n^{\prime}}^{*} b_{m} \\
& \times \exp \left[-i q\left(n-n^{\prime}\right) \Delta x_{2}\right] e^{-i q m \Delta x_{3}^{A, B}} \\
& \times e^{-i \frac{q^{2\left(n^{2}-n^{\prime 2}\right) z_{2}}}{1+\alpha z_{2}}} e^{ \pm i q x_{a}\left[\frac{\left(n-n^{\prime}\right)}{1+\alpha z_{2}}+m\right]} \\
& \times \operatorname{sinc}\left[\frac{q w}{2}\left(\frac{\left(n-n^{\prime}\right)}{1+\alpha z_{2}}+m\right)\right],
\end{aligned}
$$

where $S_{A, B}\left(\Delta x_{2}\right)$ represents both signals $S_{A}\left(\Delta x_{2}\right)$ and $S_{B}\left(\Delta x_{2}\right)$ since we included a \pm sign in Eq. (9).
The effect of defocus appears in the last three factors of Eq. (9). The first (the exponential term that depends on $n^{2}$ ) indicates that the location of the Talbot planes changes the defocus of the light source. The second (the exponential term that depends on $n$ ) indicates that there is a phase shift of the signal since the period of the self-images produced by the first grating, $G_{2}$, is not equal to that of the grating. This phase shift between signals $S_{A}\left(\Delta x_{2}\right)$ and $S_{B}\left(\Delta x_{2}\right)$ governs the measurement principle for this technique. The third (the sinc factor) indicates that the contrast of the signal decreases since the period of the optical signal is not equal to the period of the second grating $G_{3}$.

Nevertheless, the variation of the location of Talbot planes is normally very small when $\Delta z \ll f$, and it does not produce a significant variation of the signal amplitude. As is shown in Fig. 2, the signal over detector is near constant when the light source moves approximately $30 \mu \mathrm{m}$ around the focal plane. Also, the size of the photodetectors is normally much larger than the period of the grating, $w \gg p$. Then, the sinc term is significant only when the argument is close to zero, that is, when $m=-n+n^{\prime}$. Therefore, the sum over $m$ can be removed and the final expression for the signals over both detectors results in

$$
\begin{aligned}
S_{A, B}\left(\Delta x_{2}\right) \approx & \frac{I_{0}}{f^{\prime}} \sum_{n} \sum_{n^{\prime}} a_{n} a_{n^{\prime}}^{*} b_{-\left(n-n^{\prime}\right)} e^{-i q\left(n-n^{\prime}\right)\left(\Delta x_{2}-\Delta x_{3}^{A, B}\right)} \\
& \times e^{-i_{2 k}^{2}\left(n^{2}-n^{\prime 2}\right) z_{2}} e^{\mp i q x_{a} \alpha z_{2}\left(n-n^{\prime}\right)}
\end{aligned}
$$

Note that, to obtain signals $S_{A}$ and $S_{B}$, a relative displacement of grating $G_{2}$ with respect to the rest of

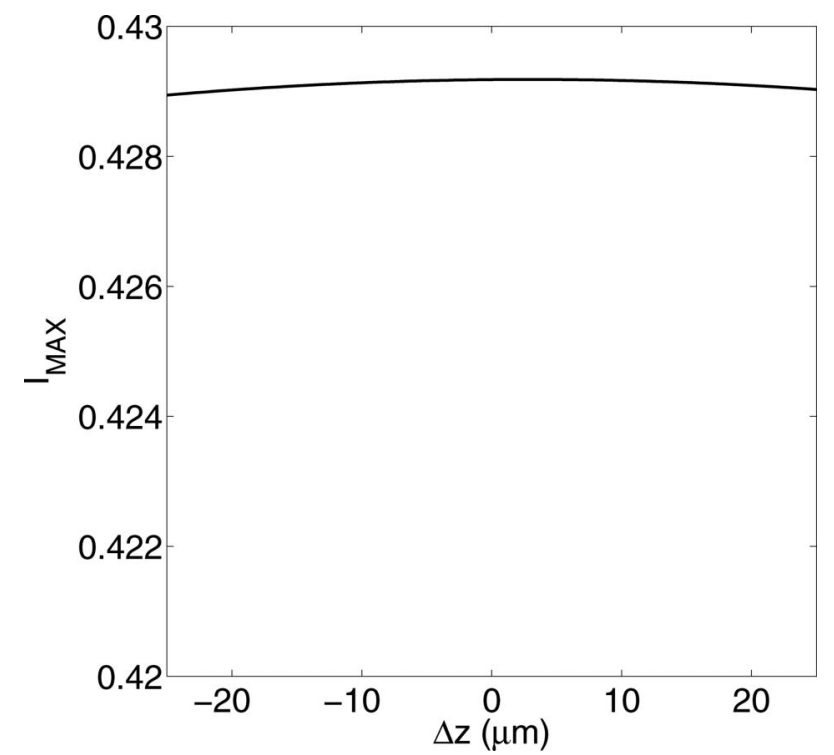

Fig. 2. Effect of defocusing: optical intensity over detector. The light source travels from $z_{0}=f-20 \mu \mathrm{m}$ to $z_{0}=f+20 \mu \mathrm{m}$. Parameters of the analysis are $p=20 \mu \mathrm{m}, \lambda=650 \mathrm{~nm}, w=1 \mathrm{~mm}$, $x_{a}=4 \mathrm{~mm}, f=10 \mathrm{~mm}, z_{1}=1 \mathrm{~mm}$, and $z_{2}=z_{T}=p^{2} / \lambda$. The intensity remains almost constant, showing the validity of our approach. 
components is required. Then there is a temporal dependence of signals with time $\Delta x_{2}=\Delta x_{2}(t)$.

The main effect of defocus is a variation in the relative phase between signals $S_{A}$ and $S_{B}$, produced by the last exponential term. This phase shift increases as the distance between the center of the photodetectors and the optical axis, $x_{a}$, increases. As a consequence, a highly sensitive collimation technique can be performed by determining the phase shift between both electrical signals, which can be measured as the distance between the maxima of both signals. This measurement can be easily automated by means of cross correlation between both signals $S_{A}\left(\Delta x_{2}\right)$ and $S_{B}\left(\Delta x_{2}\right)$ :

$$
\operatorname{corr}(f, g)=\int f(x) g(x+u) \mathrm{d} u,
$$

which can be carried out using a simple electronic circuit. Signals $S_{A}\left(\Delta x_{2}\right)$ and $S_{B}\left(\Delta x_{2}\right)$ present the same shape but are displaced from each other. Then the cross correlation presents a maximum whose position $x_{\max }$ depends on the phase shift between them. This phase shift can be obtained by $2 \pi x_{\max } / p$.

In addition, for this case the value of $\Delta x_{3}^{A}-\Delta x_{3}^{B}$ is not important; we can set it to $\Delta x_{3}^{A}-\Delta x_{3}^{B}=0$. The design of $G_{3}$ also simplifies since it can be a single diffraction grating identical to $G_{2}$.

We can analyze the signals at the detectors by using Eq. (10). As an example, let us consider that the wavelength is $\lambda=650 \mathrm{~nm}$, the grating period is $p=100 \mu \mathrm{m}$, the focal length of the collimating lens is $f=10 \mathrm{~mm}$, and both detectors have length $w=$ $1 \mathrm{~mm}$ and are placed at $x_{a}=4 \mathrm{~mm}$. The distance between components is $z_{1}=1 \mathrm{~mm}$, and $z_{2}=p^{2} / \lambda$ (the first Talbot distance) to obtain self-images of the first grating over the detectors positioned behind the second grating.

We first calculate signals $S_{A}$ and $S_{B}$, which are shown in Fig. 3; defocus $\Delta z=1 \mu \mathrm{m}$ is shown in Fig. 3(a) and defocus $\Delta z=10 \mu \mathrm{m}$ is shown in Fig. 3(b). Although it is difficult to visually appreciate a displacement between the signals along the $x$ axis, we can obtain a numerical value for this phase shift by just measuring the displacement between maximum peaks by means of correlation.

The effect of defocusing over the phase shift between signals is shown in Fig. 4. When the light source is not at focus, the signals present a displacement that can be measured by determining the phase shift between signals $S_{A}$ and $S_{B}$. In the example proposed, a defocus of $\Delta z= \pm 10 \mu \mathrm{m}$ produces a phase shift of $\pm 10^{\circ}$. In practice, due to the manufacturing process, an error of approximately $\pm 0.1 \mu \mathrm{m}$ exists in the shift between the two scanning gratings $\Delta x_{3}^{A}-\Delta x_{3}^{B}$. In the proposed example that means an error in the phase shift of $\pm 0.36^{\circ}$. In any case, this is a fixed error and it could be measured and conveniently corrected for a real device.

\section{Visual Criterion for Collimation}

When an automated procedure is not required or available but a visual criterion is enough, we can

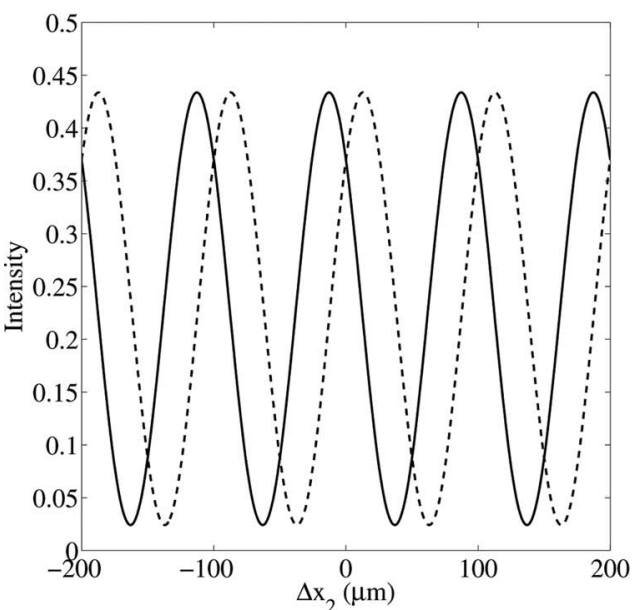

(a)

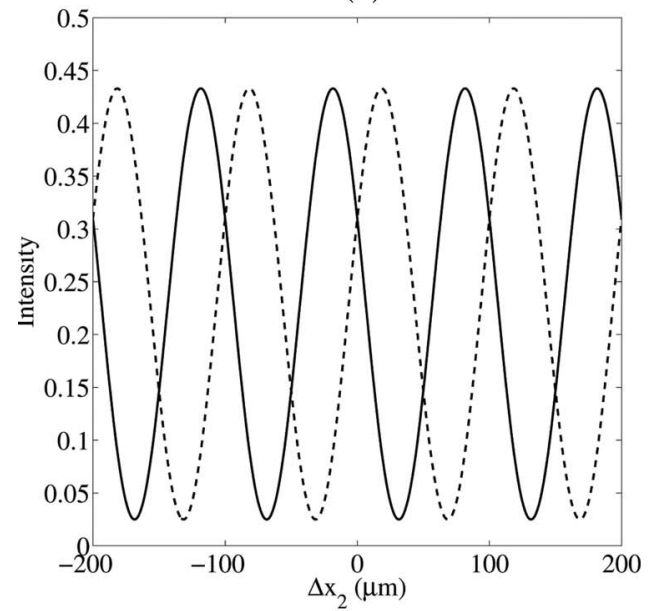

(b)

Fig. 3. Theoretical signals $S_{A}$ (solid curve) and $S_{B}$ (dashed curve) using $n=n^{\prime}=-1,0,1$ orders. The parameters for analysis are the same as in Fig. 2: (a) $\Delta z=1 \mu \mathrm{m}$ and (b) $\Delta z=10 \mu \mathrm{m}$.

use Lissajous curves produced by signals $S_{A}$ and $S_{B}$ to place the light source in the focal plane of the lens. Grating $G_{2}$ moves with respect to the rest of the components. Then there is a displacement $\Delta x_{2}$ that depends on time $\Delta x_{2}=\Delta x_{2}(t)$. This time variation produces the Lissajous figures $S_{A}\left(\Delta x_{2}\right), S_{B}\left(\Delta x_{2}\right) \rightarrow$ $S_{A}(t)$, and $S_{B}(t)$.

When the phase shift between both electrical signals is $\Delta x_{3}^{A}-\Delta x_{3}^{B}=\pi / 2$, then the Lissajous figure becomes a circle for perfect collimation. As a consequence, we set $\Delta x_{3}^{A, B}= \pm p / 4$. However, when the beam is not collimated, an additional shift is produced and then the Lissajous figure becomes an ellipse whose eccentricity depends on the shift between both signals. This dependence leads us to propose a simple visual criterion to determine the degree of collimation. As an example, Lissajous figures for different defocus distances $\Delta z$ are shown in Fig. 5 . In this case, we can visually appreciate variations in the Lissajous figure for defocus of less than $1 \mu \mathrm{m}$. Since the effect of defocusing differs depending on the sign of $\Delta z$, we can detect the direction in which we need to move the emitter. 


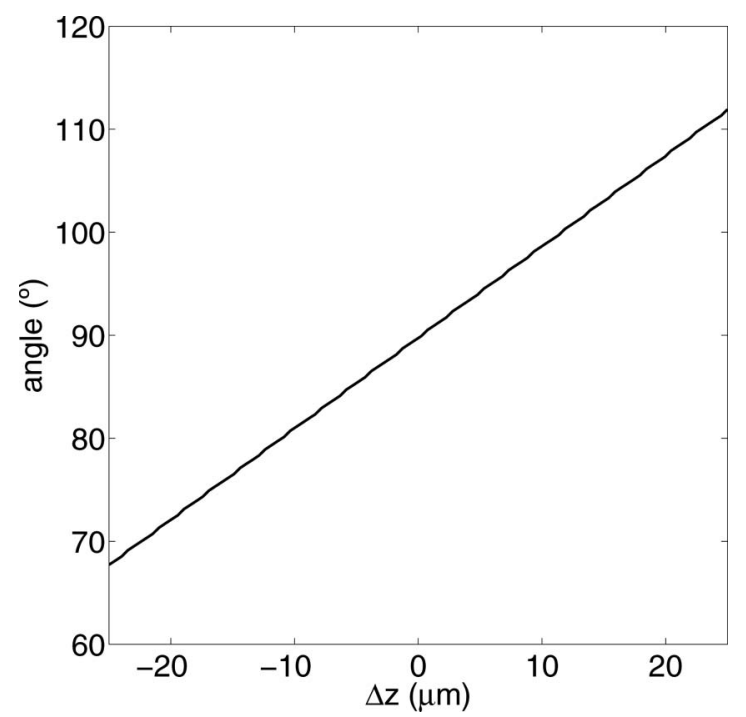

Fig. 4. Theoretical phase shift angle between $S_{A}$ and $S_{B}$ when the light source travels from $z_{0}=f-25 \mu \mathrm{m}$ to $z_{0}=f+25 \mu \mathrm{m}$. The parameters for the analysis are the same as in Fig. 2 .

\section{Experimental Results}

We performed an experiment to validate the theoretical predictions. A scheme of the setup is shown in Fig. 6. The illumination part consists of a LED (HE8 807 SG by Hitachi) with wavelength $\lambda=880 \mathrm{~nm}$ and a lens with a focal length of $f=9 \mathrm{~mm}$. The LED is positioned on a micrometric linear stage so that it can be moved with respect to the lens. The lens is placed on a metallic cylinder and the LED is displaced to ensure that it is perfectly aligned at the optical axis. The first grating $G_{2}$ is an amplitude grating with period $p=20 \mu \mathrm{m}$, and it is placed at a

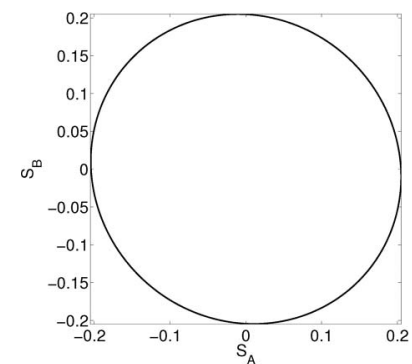

(a)

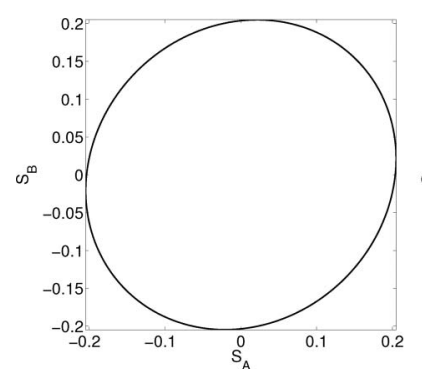

(c)

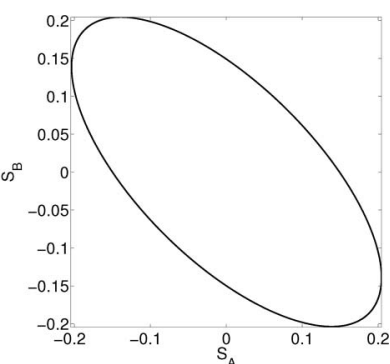

(b)

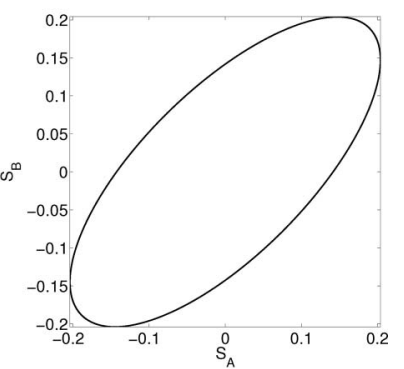

(d)
Fig. 5. Theoretical Lissajous curves using $n=n^{\prime}=-1,0,1$ orders for different light source locations: (a) $\Delta z=1 \mu \mathrm{m}$, (b) $\Delta z=10 \mu \mathrm{m}$, (c) $\Delta z=-1 \mu \mathrm{m}$, (d) $\Delta z=-10 \mu \mathrm{m}$. The rest of the parameters are the same as in Fig. $\underline{2}$.

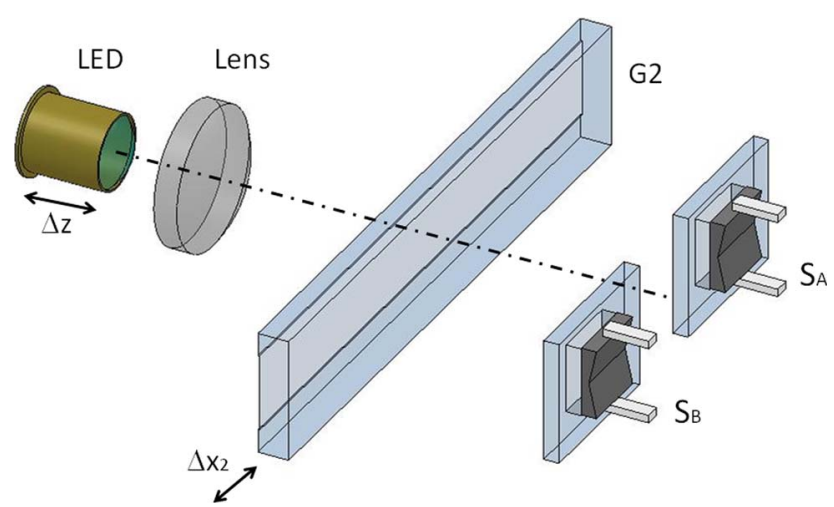

Fig. 6. (Color online) Schematic of the experimental setup.

distance $z_{1}=20 \mathrm{~mm}$ from the lens. The second grating $G_{3}$ is composed of two gratings with period $p=$ $20 \mu \mathrm{m}$ and with a lateral shift of $\Delta x_{3}^{A}-\Delta x_{3}^{B}=5 \mu \mathrm{m}$ between them. $G_{3}$ is placed at a distance $z_{2}=$ $2 p^{2} / \lambda=0.909 \mathrm{~mm}$ from the first grating. The photodetectors, with a size of $w=500 \mu \mathrm{m}$, are located directly behind the mask and are placed at $x_{a}=$ $2 \mathrm{~mm}$ from the axis.

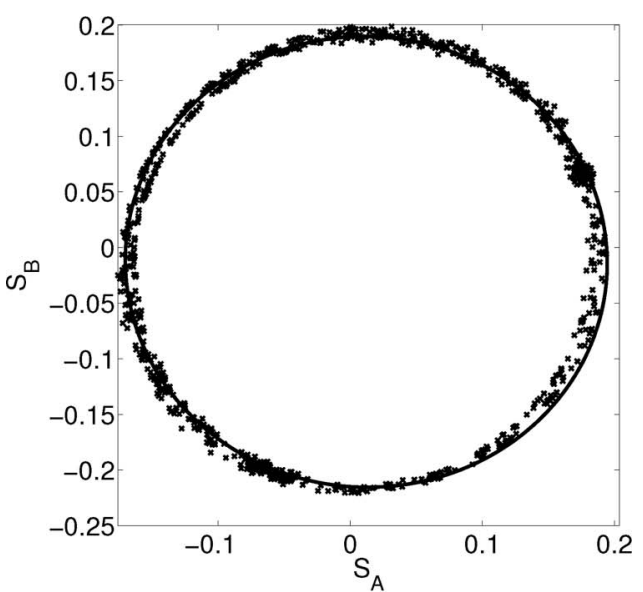

(a)

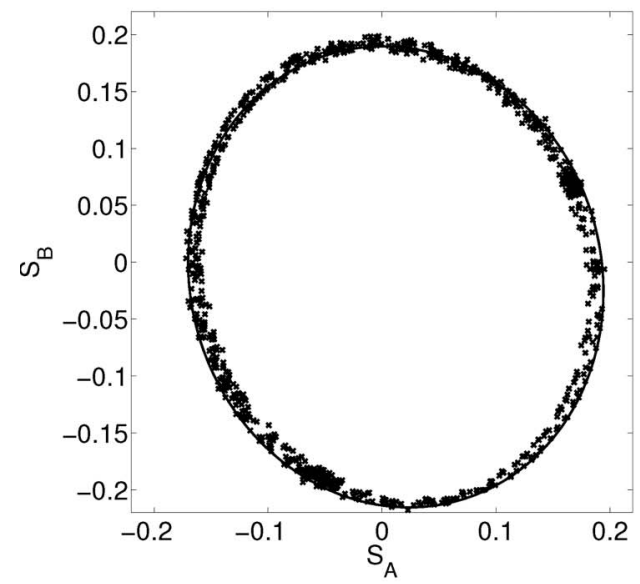

(b)

Fig. 7. Experimental Lissajous curves obtained for two different defocus distances $\Delta z$ : (a) $\Delta z=-1 \mu \mathrm{m}$ and (b) $\Delta z=10 \mu \mathrm{m}$. Crosses, experimental data; solid curve, theoretical prediction, Eq. (10). 


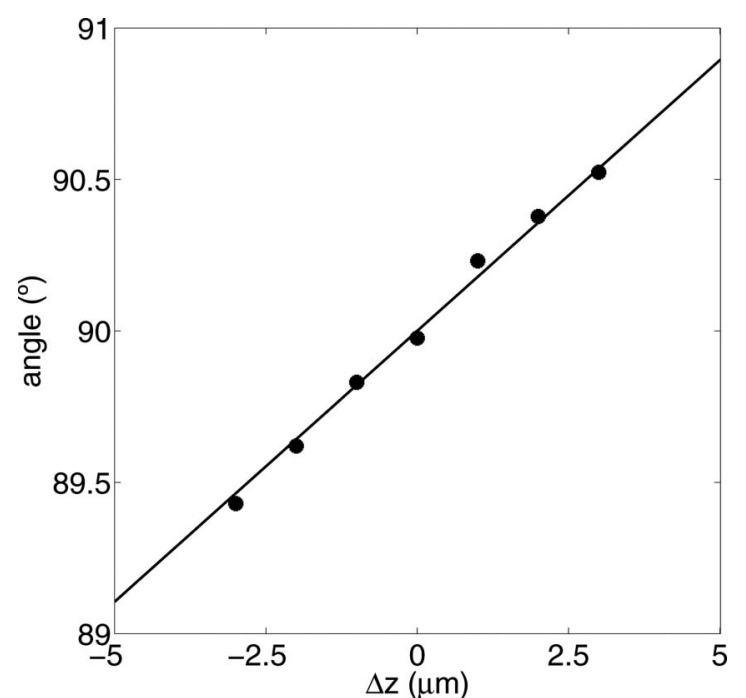

Fig. 8. Experimental phase shift angle between signals $S_{A}$ and $S_{B}$ when the light source is not at focus but is at a certain distance $\Delta z$ : circles, experimental data; solid curve, theoretical prediction.

The experiment consists of moving the first grating along the $x$ axis and acquiring the signals of the propagated intensity after the second grating. A posterior step is to perform a summation of the intensity for each photodetector separately, obtaining signals $S_{A}$ and $S_{B}$. These signals are acquired for several distances $\Delta z$ between the LED and the focal plane of the lens. As an example, the experimental Lissajous figures (crosses) for $\Delta z=-1 \mu \mathrm{m}$ and $\Delta z=10 \mu \mathrm{m}$ are shown in Fig. 7, where a variation in the shape is observed. In addition, in Fig. 7 we include the theoretical prediction (solid curve) given by the theoretical expressions using the experimental values for all the variables. The experimental Lissajous figures appear off-center since one of the two photodetectors collected more light than the other. We then centered the theoretical prediction to emphasize the almost perfect concordance between theory and experiment.

We also determined the phase shift between signals $S_{A}$ and $S_{B}$ in terms of $\Delta z$ using a correlation technique, as is shown in Fig. 8. We can see that, for a displacement of $\Delta z=5 \mu \mathrm{m}$, there is a phase shift of $1^{\circ}$, which can be easily detected. Since resolutions of $0.1^{\circ}$ in the determination of the phase shift of two sinusoidal signals can be achieved, we could obtain a resolution of approximately $0.5 \mu \mathrm{m}$ by placing the LED in the focal plane of the lens.

\section{Conclusions}

We have presented a technique to place a light source in the focal plane of a lens to obtain a collimated light beam. The technique is based on a double grating system and consists of measuring the phase shift produced in the signals acquired with two photodetectors that are off axis. A Fresnel approach is used to obtain the analytical expressions that describe the system. Also, experimental results show the va- lidity of the technique. The sensitivity of the method is quite good in comparison with conventional collimation methods. Displacements of $\Delta z \approx 1 \mu \mathrm{m}$ are detectable.

This research has been supported by project CCG08-UCM/DPI-3952 of Dirección General de Universidades e Investigación de la Consejería de Educación de la Comunidad de Madrid and by project DPI2008-02391 of the Ministerio de Ciencia e Innovación of Spain.

\section{References}

1. J. Jahns and A. W. Lohmann, "The Lau effect (a diffraction experiment with incoherent illumination)" Opt. Commun. 28, 263-267 (1979).

2. K. Patorski, "The self-imaging phenomenon and its applications," Prog. Opt. 27, 3-108 (1989).

3. S. C. Som and A. Satpathi, "The generalized Lau effect," J. Mod. Opt. 37, 1215-1226 (1990).

4. L. Liu, "Partially coherent diffraction effect between Lau and Talbot Effects," J. Opt. Soc. Am. A 5, 1709-1716 (1988).

5. K. V. Avudainayagam and S. Chitralekha, "Lau effect and beam collimation,” J. Mod. Opt. 44, 175-178 (1997).

6. K. V. Avudainayagam and S. Chitra Nayagam, "Two-grating diffraction and Lau effect under laser illumination," Appl. Opt. 36, 2029-2033 (1997).

7. Ch. Siegel, F. Loewenthal, and J. E. Balmer, "A wavefront sensor based on the fractional Talbot effect," Opt. Commun. 194, 265-275 (2001).

8. C. L. Hou and J. Bai, "Wavefront measurement for long focal large aperture lens based on Talbot effect of Ronchi grating," J. Phys.: Conf. Ser. 48, 1037-1041 (2006).

9. S. Chang, "Geometrical aberrations of self.imaged line gratings," Optik (Jena) 116, 379-389 (2005).

10. S. Yokozeki and K. Ohnishi, "Spherical aberration measurement with shearing interferometer using Fourier imaging and moiré method," Appl. Opt. 14, 623-627, (1975).

11. K. Patorski, S. Yokozeki, and T. Suzuki, "Collimation test by double grating shearing interferometer," Appl. Opt. 15, 12341240, (1976).

12. J. P. Bétend-Bon, L. Wosinski, and M. Breidne, "Double grating phase stepping interferometry for testing aspherics," Pure Appl. Opt. 1, 55-69 (1992).

13. J. C. Bhattacharya and A. K. Aggarwal, "Measurement of the focal length of a collimating lens using the Talbot effect and the moiré technique," Appl. Opt. 30, 4479-4480 (1991).

14. S. Chang and S. I. Lee, "First-order aberration of a misfocused self-imaging system," Optik (Jena) 119, 742-748 (2008).

15. S. Rana, S. Prakash, and S. Prakash, "Automated collimation testing in Lau interferometry using phase shifting technique," Opt. Lasers Eng. 47, 656-661 (2009).

16. D. Joeux and Y. Cohen-Sabban, "High magnification selfimaging," Appl. Opt. 21, 625-627 (1982).

17. Y. Cohen-Sabban and D. Joyeux, "Aberration-free nonparaxial self-imaging," J. Opt. Soc. Am. 73, 707-719 (1983).

18. S. Szapiel and K. Patorski, "Fresnel diffraction images of periodic objects under Gaussian beam illumination," Opt. Acta 26, 439-446 (1979).

19. L. M. Sanchez-Brea, J. Saenz-Landete, J. Aloso, and E. Bernabeu, "Invariant grating pseudo-imaging using polychromatic light and finite extension source," Appl. Opt. 47, 1470-1477 (2008). 